\title{
Cuantificación y caracterización de las comunidades marginadas y aisladas de Colombia, Ecuador y Perú
}

\section{Quantification and characterization of the communities isolated and marginalized of Colombia, Ecuador and Peru}

Susana Herrero Olarte* sherrero@udla.edu.ec

\begin{abstract}
Resumen
Las Comunidades Marginadas y Aisladas (CMA) en América Latina, y en concreto en los Andes, presentan unas características propias que generan la necesidad de diseñar políticas para mejorar sus condiciones de vida. Con el objetivo de definir su situación, en este artículo apoyado en fuentes de información primarias y secundarias, se definen y cuantifican las CMA andinas en los tres países. A continuación se presentan datos sociodemográficos fundamentales comparados de las CMA con las medias nacionales. En todos los casos se registran indicadores similares entre las CMA pero sustancialmente diferentes al compararlos con los promedios nacionales, siempre muy por encima.
\end{abstract}

\section{Palabras clave}

América Latina, Andes, desarrollo rural, comunidades marginadas y aisladas.

\section{Abstract}

Isolated and Marginalized Communities (CMA) in Latin America, and particularly in the Andes, have some characteristics that generate the need to design policies to improve their living conditions. In order to define their situation, this paper relied on primary and secondary sources of information are defined and quantified the CMA in the three Andean countries. Then basic demographic data of the CMA compared with national averages are presented. In all cases similar indicators are reported among CMA but substantially different when compared with national averages, always above.

\section{Keywords}

Latin America, Andes, Rural development, Isolated and Marginalized Communities (CMA).

* Phd en Economía Aplicada. Directora del Centro de Investigaciones. Facultad de Ciencias Económicas y Empresariales. Universidad de las Américas, Quito- Ecuador. 

las comunidades marginadas y aisladas de Colombia, Ecuador y Perú. Revista Retos, 10(2), pp.135-146.

\section{Introducción}

En América Latina se comparten necesidades y problemáticas comunes entre varios países, que cuentan con historias parecidas y para los que tentativamente podrían plantearse estrategias de resolución también comunes. Uno de los retos fundamentales es cómo abordar la situación de retroceso que padecen las personas que viven en las Comunidades Marginadas y Aisladas (CMA). Para ello es fundamental identificarlas y reconocer sus principales características demográficas. Las características se ven altamente condicionadas por la región natural latinoamericana en la que se ubiquen (Andes, selva, zona árida, etc.) Por ello, para contextualizar su realidad de cara a diseñar políticas para mejorar sus condiciones de vida, es preciso diferenciar y analizar las CMA por zonas naturales.

En este caso se van a tomar tres países, Colombia, Ecuador y Perú y se van a definir y cuantificar las personas que viven en CMA. A continuación se acotará el análisis a las comunidades andinas. La información primaria y secundaria de las comunidades permitirá realizar un análisis comparado entre su realidad y la nacional. Se pretende comprobar hasta qué punto las condiciones de vida son mejores o peores en las CMA. Los datos utiliza- dos serán la tasa de natalidad la tasa de fertilidad la esperanza de vida la tasa de mortalidad anual la pirámide poblacional por edades y sexo, los hogares encabezados por una mujer y la tasa de inactividad por edades.

\section{Metodología}

En el ámbito agrícola, la condición de marginalidad se asocia con la imposibilidad para generar los recursos económicos y producir los alimentos necesarios en el corto, medio y largo plazo, lo que viene a perpetuar estructuras que tienen como fin la supervivencia. Efectivamente, desde un punto de vista agrícola las áreas marginales se definieron por Sebastian (2009) como aquellas tierras en países de ingreso medio y bajo ${ }^{1}$ en las que los niveles de productividad son inferiores a la media (planicies de altitud, las colinas acusadas, tierras bajas accidentadas, zonas áridas o semiáridas, etc.) y que son rurales. Las tierras en condición de aislamiento para Sebastian eran las que estaban a más de dos horas del mercado más cercano.

1 Son países de ingreso bajo los que perciben sus habitantes 1035 dólares o menos al año, entre 1036 y 4085 son países de ingreso medio bajo, los países de ingreso medio alto perciben entre 4086 y 12615 dólares, y el ingreso por habitante de los países de ingreso alto es igual o superior a 12616 (Banco Mundial, 2015). 
En este trabajo, para cuantificar las personas que viven en condición de marginación en Colombia, Ecuador y Perú es preciso analizar si se trata de países de ingreso medio o bajo, las zonas en las que no se alcanzan, por su naturaleza, niveles óptimos de productividad agropecuaria $y$, dentro de éstas, las áreas rurales.

Colombia, Ecuador y Perú son países de ingreso medio alto, según el Banco Mundial, mientras según el Comité de Ayuda al Desarrollo, son países en desarrollo, de ingreso medio alto (CEPAL, 2012).

En los tres países, pueden considerarse zonas poco aptas para la producción agropecuaria la selva, la región andina, y las zonas áridas. Juntas suman el $74,2 \%$ del territorio colombiano, el $93 \%$ del ecuatoriano y el $98 \%$ del peruano.

La selva es una zona poco apta para la producción agropecuaria por los bajos niveles de productividad por la alta acidez de la tierra, los altos niveles de aluminio y los bajos registros de fósforo, potasio y material orgánico (Sánchez, et al., 1982) que se traducen en el reconocimiento del $6 \%$ de la tierra de la selva como apta para la producción agropecuaria continua y el $7,5 \%$, que podría serlo aplicando sistemas de rotación simples (ONERN, 1981).

La zona andina es una zona poco apta por las pendientes. La cordillera de los Andes atraviesa a Colombia y se ramifica en tres cordilleras continentales y una cordillera sumergida en el océano Pacífico. La cordillera Central es la más alta con una altura de 5750 metros, mientras la occidental alcanza los 4280 metros, y la oriental llega a los 5000 (Melo, 2004). La zona montañosa ecuatoriana se divide en dos cordilleras que oscilan entre los 3500 y los 4000 metros, con el Chimborazo como el nevado más alto, alcanzando los 6310 metros, y el Cayambe, con 5790 (Campaña, 2009). La cordillera de los Andes atraviesa la sierra peruana de sur a norte y se encuentra formada tres cordilleras: la cordillera Occidental, que tiene el Nevado Huascarán, considerado el más alto con 6768 metros, la Central, que alcanza con el Salccantay los 6271 metros, y la cordillera Oriental, que con el nevado Auzangate, llega a los 6384 metros (Morales, 1998).

Las zonas áridas se caracterizan por el escaso contenido de carbono (Lal, 2002) que se traduce en una duración de la estación de crecimiento menor a 119 días, cultivándose de manera general pasto, miso, sorgo y ajonjolí (FAO, 1993). De hecho, en 1980 el 41\% de la tierra árida del mundo se dedicaba al pastoreo, el $25 \%$ a las granjas, y el $16 \%$ no se utilizaban (Heathcote, 1983). En el presente análisis se consideran tierras áridas aquéllas que se clasifican como semiáridas, áridas, hiperáridas y xéricas. Una tierra es árida cuando tiene al menos siete meses secos, un índice de aridez inferior a 0,5 y cuando la pluviosidad es menor a $500 \mathrm{~mm}$ 
en invierno y a $600 \mathrm{~mm}$ en invierno (FAO, 1993 y Verbist et al., 2010).

Dentro de lo que son las áreas marginales es necesario considerar las personas que viven en el ámbito rural en cada una de estas áreas. En Colombia, la población rural es la que vive en poblados de menos de 1500 habitantes y la población dispersa. En Ecuador, son las personas censadas en la cabecera parroquial definida como rural, en el resto de la parroquia, y las empadronadas de la periferia de las capitales provinciales y cabeceras cantonales. En Perú son las personas que viven entre los linderos de los centros urbanos (CEPAL, 1999). En Colombia el 54\% de la población que vive en la selva vive en el ámbito rural, en Ecuador el 61\% y en Perú el 44\%. En los Andes vivía en el ámbito rural en Colombia el 23\% de la población, en Ecuador el $43 \%$ y en los andes peruanos el $54 \%$ del total. En tanto a las zonas áridas, en Colombia el 15\%, en Ecuador el 33\% y en Perú el $15 \%$.

Equipararemos la dispersión con la dispersión con la ruralidad, y consideraremos las familias que viven en el ámbito rural en las zonas marginales se encuentran en condición de dispersión. Esto supone 20828296 personas en total, y en la región andina 13554783 (Oficina Nacional de Evaluación de Recursos Naturales, 1981, Centro de Investigaciones de Zonas Áridas de Perú, 2006, Matamoros, 2007, Rodríguez, 2007, FLACSO-MAE-PNUMA, 2008,
Departamento Administrativo $\mathrm{Na}$ cional de Estadística de Colombia, 2010, Instituto Nacional de Estadísticas y Censos de Ecuador, 2010, Ministerio del Ambiente de Perú, 2010, Verbist et al., 2010, Ministerio del Ambiente de Ecuador, 2010, Ministerio de Defensa Perú, 2011, Instituto de Estadísticas e Informática de Perú, 2015, y Molano, 2015).

Para conocer la realidad de las CMA en la región andina se atendió a información primaria y secundaria sobre la realidad de las comunidades en Colombia, Ecuador y Perú entre 2009 y 2014. Para obtener la información primaria se visitaron cincuenta comunidades andinas en los tres países de la mano de una organización colombiana, cinco ecuatorianas, y una organización peruana, entrevistándose a 36 directivos o técnicos de las organizaciones. Se realizaron 216 encuestas individuales a las familias que viven en las CMA y 170 personas participaron en entrevistas en grupo. En total se entrevistaron, individualmente y en grupo, 40 familias que residen en CAM andinas en Colombia, 307 en Ecuador, y 39 en Perú. En tanto a la información secundaria, se utilizaron las líneas de base o diagnósticos iniciales de ocho proyectos de cooperación al desarrollo llevados a cabo en CAM andinas en la región andina que abarca Colombia, Ecuador y Perú. Los resultados se compararon con las medias nacionales, obtenidas del Banco Mundial (2015). 


\section{Resultados}

A continuación se analizan de manera comparada entre las CMA y los promedios nacionales la esperanza de vida, la tasa de mortalidad, la forma de la pirámide poblacional, la cantidad de mujeres, los hogares encabezados por mujeres, la tasa de fertilidad, los hijos por familia y la tasa de inactividad por edades.

Al considerar la esperanza de vida al nacer se evidenciaban marcadas diferencias entre los países en estudio, tanto entre la media nacional como en las CMA. Ecuador tenía la mayor esperanza de vida al nacer al- canzado 75 años a nivel nacional, seguida de cerca por Colombia y Perú con dos años menos, es decir 73 años de edad. La esperanza de vida al nacer en las CMA de Colombia era de 50 años, menor que las de Ecuador, de 53 años, y Perú, de 56 años. La diferencia entre la esperanza de vida al nacer entre las CMA y la media nacional era amplia, como se indica a continuación. Colombia era el país que mayor diferencia presentaba con 23 años, seguido por Ecuador con una diferencia de 22 años, mientras que Perú tiene una diferencia de 17 años.

\section{Figura 1. Esperanza de vida al nacer en número de años}

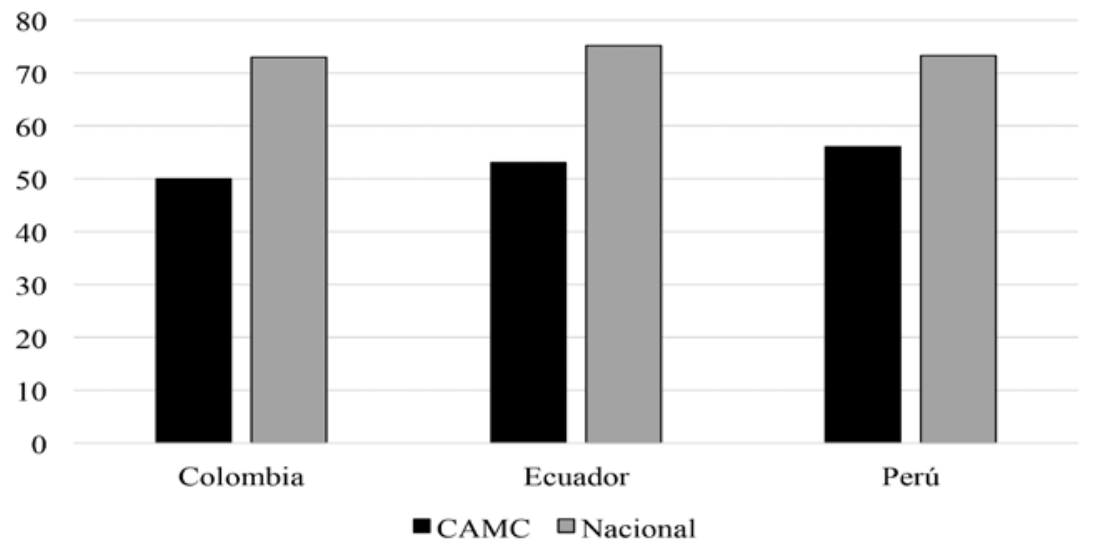

Fuente: Entrevistas en las CMA y líneas de base de proyectos de cooperación y Banco Mundial (2015)

Profundizando el tema, si se consideraba la tasa de mortalidad anual, se evidenciaba que en las CMA la tasa de mortalidad casi duplicaba la media nacional en los tres países analizados, como se indica a continuación. El caso más extremo es el de Perú donde la media nacional era de cinco por cada 1000 personas y la tasa de las CMA era de 11 por cada 1000 personas. Por su parte, Colombia reportaba una tasa de mortalidad nacional de seis por cada 1000 personas, la más alta de los tres países, 
sin embargo la tasa de mortalidad en las CMA era de 10 por cada 1000 personas, superior a la de la media nacional. El caso de Ecuador era similar: la tasa media nacional de mortalidad era de cinco por cada 1000 personas y la tasa de mortalidad de las CMA de nueve por cada 1000 personas.

Figura 2. Tasa de mortalidad anual por cada 1000 personas

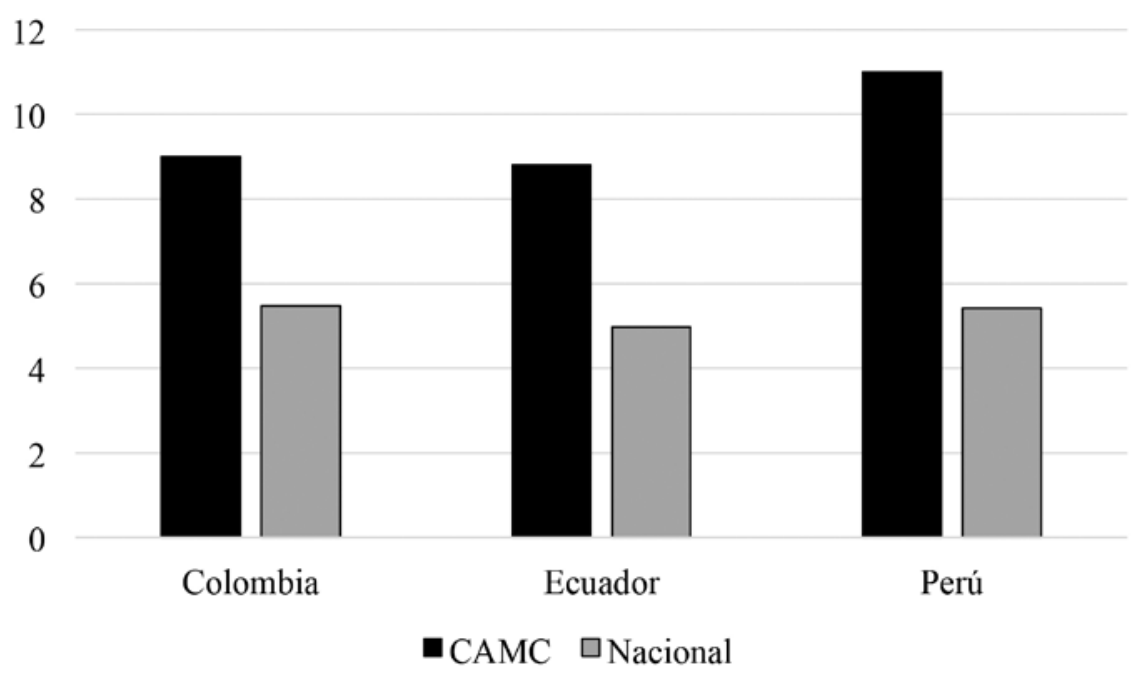

Fuente: Entrevistas en las CMA y líneas de base de proyectos de cooperación y Banco Mundial (2015)

Examinando la estructura de la población se evidenciaba que las CMA se caracterizaban por tener una alta población joven, como se indica en la figura 3. Si se considera la población entre cero y 14 años se puede evidenciar que ésta representaba más de la mitad de la población total en los tres países en estudio.
Ecuador reportaba el mayor porcentaje alcanzado $65 \%$ de la población total, frente al 55\% de Colombia y al $61 \%$ de Perú. Comparando con la media nacional se puede evidenciar que la diferencia entre las CMA y la media nacional era muy marcada, sobre todo en Ecuador y Perú. 
Figura 3. Población entre 0 y 14 años como porcentaje del total

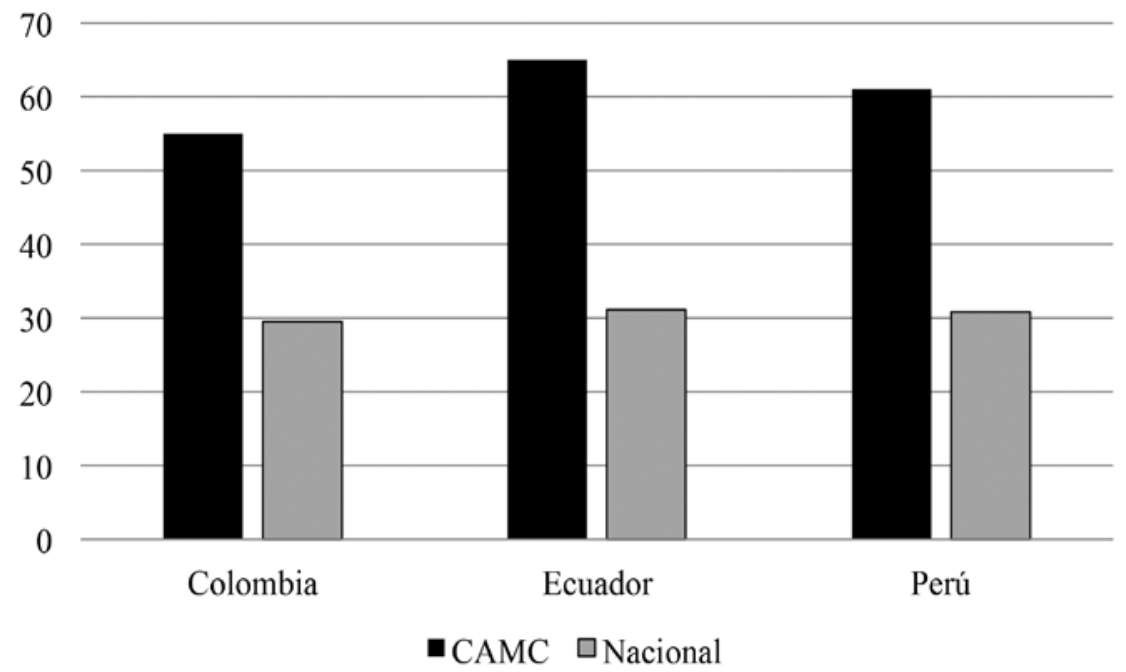

Fuente: Entrevistas en las CMA y líneas de base de proyectos de cooperación y Banco Mundial (2015)

Sin embargo, no se evidencia una diferencia tan amplia entre la media nacional de los países en estudio y las comunidades en la población mayor a 65 años. La mayor diferencia se podía apreciar en Colombia que reportaba una media nacional para la población mayor a 65 años de $8 \%$ mientras que la de las CMA era del 5\%. En Ecuador y Perú esa diferencia era de dos puntos porcentuales, ubicándose la media nacional para los dos países en el $6 \%$, por debajo de la de las CMA.
Una importante característica de las CMA es el porcentaje de mujeres frente al total de la población, como se indica a continuación. La media nacional en los tres países analizados es cercana al 50\% una amplia diferencia frente a las comunidades. En las CMA más del $60 \%$ de la población era femenina, una diferencia de por lo menos 10 puntos porcentuales frente a las medias nacionales. Así, en Colombia el 67\% de la población en las CMA son mujeres, en Ecuador el $60 \%$ y en Perú el $61 \%$. 
Figura 4. Porcentaje de mujeres

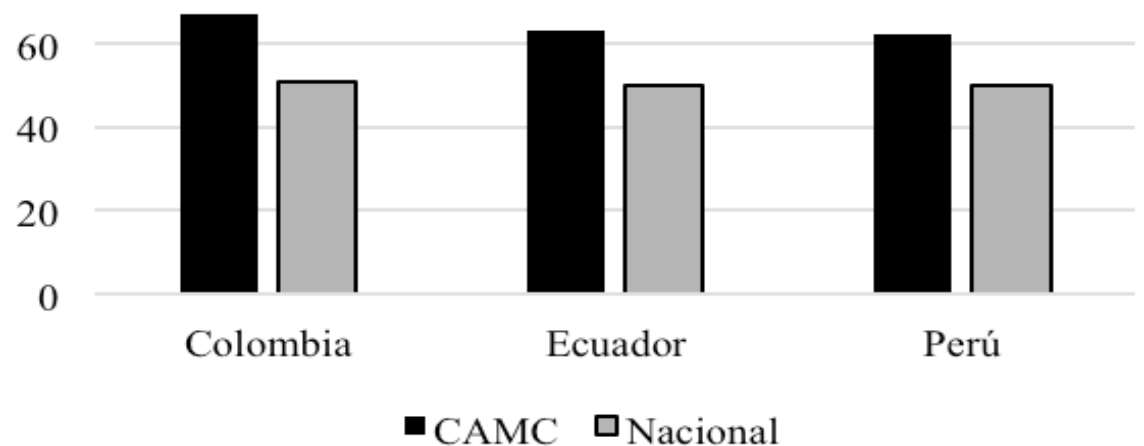

Fuente: Entrevistas en las CMA y líneas de base de proyectos de cooperación y Banco Mundial (2015)

Además de la alta proporción de mujeres en las CMA, destaca el número de mujeres jefe de hogar. En este aspecto, se identifican marcadas diferencias no sólo entre las medias nacionales y de las CMA sino entre los tres países en estudio, como se indica en la figura 5. El porcentaje de mujeres jefes de hogar en Colombia a nivel nacional es de $34 \%$, muy por encima de lo reportado en Ecuador
(20\%) y Perú (23\%). Además, en el caso de las comunidades colombianas, el 75\% de los hogares son encabezados por una mujer, lo cual implica una importante diferencia frente a la media nacional y a lo reportado en las CMA en Ecuador (60\%) y Perú $(65 \%)$. A pesar de que no alcanzaban los niveles de Colombia, seguían superando en casi tres veces la media nacional de su respectivo país.

\section{Figura 5. Hogares encabezados por mujeres como porcentaje del total}

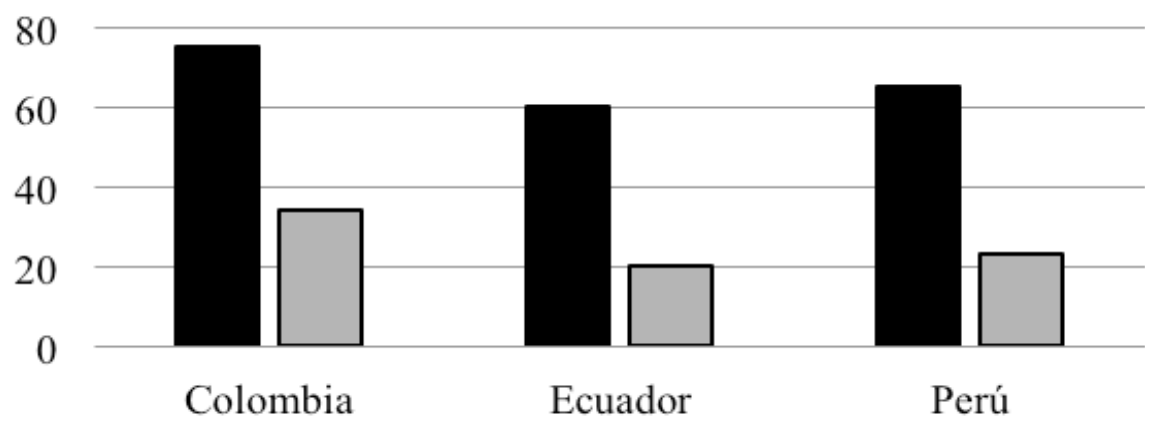

- CAMC $\square_{\text {Nacional }}$ 
Analizando la tasa de fertilidad se puede ver que las CMA reportan una mayor tasa de fertilidad frente a la media nacional de sus respectivos países. Las comunidades tenían una tasa de fertilidad de cinco nacimientos por mujer, en el caso de Colombia y Ecuador, y de cuatro nacimientos por mujer en Perú. Por su parte, la tasa media nacional de fertilidad en Colombia era de dos nacimientos por mujer, mientras que en Ecuador y Perú era de tres nacimientos por mujer.

Desagregando la información de fertilidad por grupos de edad, se puede evaluar la problemática de las madres adolescentes, que son aquellas mujeres de entre 15 y 19 años que se encontraban embarazadas o tenían hijos al momento de la entrevista. Se pone en evidencia que la media nacional para Colombia, Ecuador y Perú alcanzaba en promedio $18 \%$, mientras que la tasa de las CMA en los dos países era significativamente más alta alcanzando en promedio $40 \%$. Es decir, las madres en las CMA en promedio empiezan la gestación a muy temprana edad lo cual explicaría, al menos en parte, la diferencia en la tasa de fertilidad.

\section{Conclusiones}

Las Comunidades Marginadas y Aisladas (CMA) desde un punto de vista agrícola son aquellas en las que es difícil producir alimentos y se encuentran en condición de dispersión. En Colombia, Ecuador y Perú, por su holografía y el volumen de población rural, viven casi 21 millones de personas en las CMA. Más de 13 millones y medio viven en las CMA en los Andes, lo que permite definir y caracterizar mejor sus condiciones de vida, por las peculiaridades del territorio, y diseñar políticas que puedan mejorar sus condiciones de vida. Para lograrlo es necesario conocer sus características básicas como grupo, e identificar cuáles son sus principales problemas.

Examinando algunos indicadores demográficos fundamentales de Colombia, Ecuador y Perú y las CMA que se asientan en sus territorios se evidencian importantes diferencias vinculadas a los principales indicadores de desarrollo humano, que lastran su avance. Las familias en las comunidades viven veinte años menos y su tasa de mortalidad duplica al promedio nacional. Es una población eminentemente joven, con un volumen de niños y jóvenes hasta los 14 años que supera en 30 puntos el promedio nacional. La diferencia es sustancialmente menor al valorar la población mayor, evidenciándose una brecha entre la población económicamente activa a nivel nacional, y la que se registra en las CMA. Dado que parte de la población capaz de generar ingresos migrando de manera temporal o por periodos, queda en las comunidades un mayor porcentaje de mujeres, tradicionalmente vinculadas al cuidado de la familia y el hogar mientras el hombre trabaja. En la medida en que el hombre puede continuar o no vinculado a la familia, se registran 
más del doble de hogares encabezados por mujeres. Sin acceso a los programas de planificación familiar, las mujeres tienen más hijos y los tienen antes pese a la evidente dificultad para mantenerlos.

Se observan diferencias más que significativas entre los promedios registrados en las CMA y las medias nacionales. Entre las CMA de tres países se aprecian también diferencias aunque en ningún caso resultan significativas, lo que muestra cómo la condición de marginación y el aislamiento son causas fundamentales y definitivas de la ralentización de sus indicadores de calidad de vida.

Efectivamente, la dificultad para la producción agropecuaria viviendo en el campo y la condición de aislamiento invita a la sociedad a avanzar de manera independiente de ellos y a las autoridades y a los organismos internacionales a aparcar el trabajo con este tipo de comunidades porque es difícil generar iniciativas viables y sostenibles. En consecuencia, los niveles de pobreza, inseguridad alimentaria y de falta de educación y salud resultan preocupantes especialmente para las comunidades, requiriéndose políticas especialmente diseñadas para ellos, capaces de generar cambios reales sobre sus perspectivas de vida.

\section{Bibliografía}

Banco Mundial (2015). Base de datos del BM. Banco Mundial, Washington. Recuperado de http://datos.bancomundial.org/ el 27 de marzo de
2015

Centro de Inteligencia Americano, CIA (2014). The World Fact book. CIA, Washington. Recuperado de www. cia.gov/library/publications/theworld-factbook/fields/2046.html el 20 de mayo de 2015.

Centro de Investigaciones de Zonas Áridas de Perú (2015). Líneas de investigación del presente. Universidad Nacional Agraria la Molina: Lima. Recuperado de http://www.lamolina.edu.pe/ciza/ el 27 de abril de 2015

Comisión Económica para América Latina, CEPAL, 1999. América Latina: proyecciones de población urbano rural. Boletín Demográfico No. 63, de enero de 1999 http://www.cepal.org/celade/ publica/bol63/bd63def00e.html el 20 de marzo de 2015

Comisión Económica para América Latina, CEPAL (2012). Panorama Social de América Latina.

CEPAL, Santiago de Chile. Recuperado: www.cepal.org/es/publicaciones /1247-panorama-social-de-america-latina-2012 el 20 de abril de 2015.

FAO (1993). Desarrollo sostenible de tierras áridas y lucha contra la desertificación. FAO, Roma.

FLACSO-MAE-PNUMA (2008). GEO Ecuador 2008 informe sobre el estado del medio ambiente. FLACSO, Quito. Recuperado de http://www. flacsoandes.edu.ec/libros/digital/41444.pdf el 16 de marzo de 2015

Heathcote, R. L. (1983). The arid lands: Their use and abuse. Londres: Longman.

Instituto de Estadísticas e Informática de Perú (2005). Tasa de crecimiento de la población por departamento. IESI, 
Lima. Recuperado de http://proyectos.inei.gob.pe/web/biblioineipub/bancopub/Est/Lib0005/cap-52. htm el 27 de abril de 2015 (2006). Censo nacional de población y vivienda 2005. IESI, Lima. Recuperado de http://censos.inei.gob.pe/ Censos2005/redatam/\# el 20 mayo de 2015.

Instituto Nacional de Estadísticas y Censos de Ecuador (2010). Censo población y vivienda 2010. INEC, Quito. Recuperado de http://www.ecuadorencifras.gob.ec/censo-de-poblacion-yvivienda/ el 16 de abril de 2015

Lal, R. (2002). Carbon sequestration in dryland ecosystems of west Asia and North Africa. Land. DEg. 13, 45-49

Matamoros, A, Coord (2007). Documento sobre gestión de la biodiversidad amazónica en ecuador, Programa OTCA - BID ATN/OC - 9251 - RG. Recuperado de: http://www.otca.info/ biodiversidade/2009/publico/_arquivos/File/Documento\%20Ecuador\%20(final).pdf el 20 de mayo de 2015

Melo, V. (2004). Nuevo Identidades 5: Sociales. Norma, Bogotá. Recuperado de https://books.google.com.ec/bo oks?id=peN2WXwJfHgC\&pg=PA $21 \& \mathrm{lpg}=\mathrm{PA} 21 \& \mathrm{dq}=$ relieve + colo mbiano + cordilleras \& source $=b l \&$ ots $=Z f S \_31 z \_$Oz\&sig=T2HaBtHw GbBF4SSkICXMmk7x8Ww\&hl= es-419\&sa $=$ X\&ei $=3 \mathrm{Wpj}$ VeqIOO3 dsASHsYLQBQ\&ved=0CGcQ6A $\mathrm{EwDg}_{\mathrm{w}} \mathrm{\textrm {v }}=$ onepage $\& \mathrm{q}=$ relieve $\% 20$ colombiano $\% 20$ cordilleras $\& \mathrm{f}=$ fals e el 16 de abril de 2015

Morales, B. (1998). Satellite Image Atlas of Glaciers of the World South America. Gobiernos de los EEUU, Washington. Recuperado de http://pubs. usgs.gov/pp/1386i/report.pdf el 27 de marzo de 2015
Ministerio de Defensa Perú (2011). Libro Blanco de la defensa Nacional, Capítulo II. Recuperado de http://www. mindef.gob.pe/menu/libroblanco/ pdf/Capitulo_II.pdf el 16 de mayo de 2015

Ministerio del Ambiente de Ecuador (2010). Página web. Recuperado de https://www.google.com. ec/?gfe_rd=cr\&ei=owQ4 Va2 QLrOw $8 w f r 4 I D A A g \& g w s$ $\mathrm{rd}=\mathrm{ssl} \# \mathrm{q}=$ zonas + aridas + peru\& sfl=function $\% 20$ ()\%20\{try\{var\%20 _0x57571111 el 20 de abril de 2015 Ministerio del Ambiente de Perú (2010). Zonas Áridas, semiáridas, subhúmedas y degradación de tierras en el Perú. Ministerio del Ambiente de Perú, Lima. Recuperado de https://www. google.com.ec/?gfe_rd=cr\&ei=ow Q4Va2QLrOw8wfr4IDAAg\&gws_ $\mathrm{rd}=\mathrm{ssl} \# \mathrm{q}=$ zonas + aridas + peru\& sfl=function $\% 20() \% 20\{$ try\{var\%20 _0x5757 el 27 de marzo de 2015

Molano, J. (2015). Zonas áridas de Colombia. Contribución al estudio de su geografía económica y humana. Sociedad geográfica de Colombia. Academia de ciencias geográficas, Bogotá. Recuperado de http://www.sogeocol.edu. co/documentos/084_zon_arid_de_ col.pdf el 7 de mayo del 2015.

Oficina Nacional de Evaluación de Recursos Naturales (1981). Inventario nacional de tierras del Perú. Lima: ONERN.

Rodríguez, A. (2007). Seminario taller sobre la sinergia de los operativos censales. Grupo de trabajo FAO/OEA-CIE/ IICA, Estadísticas Agrícolas y Ganaderas para América Latina y el Caribe. FAO, Ciudad de México. Recuperado de: http://www.inegi. org.mx/inegi/contenidos/espanol/ eventos/sinergia/12_dic/A_Rodriguez_Colombia.pdf el 16 de marzo de 2015 
Sánchez, P.A.; D.E. Bandy; J.H. Villachica, \& J.J, Nicholaides (1982). Amazon Basin Soils: Management for Continous Crop Production. Science, 216, 821-827.

Sebastian, K. (2009). Mapping favorability for agriculture in low and middleincome countries: technical report, maps and statistical tables. Washington: Oxfam America.
Verbist, K., Santibañez, F., Gabriels, D., \& Soto, G. (2010). ATLAS de Zonas Áridas de América Latina y el Caribe. UNESCO-PHI-CAZALAC, Montevideo. Recuperado de http:// www.desertificacion.gob.ar/wpcontent/uploads/2013/04/Atlas_ de_Zonas_Aridas_de_ALC_Espanol.pdf el 20 de mayo de 2015

Recepción: 07-09-2015 - Aceptación: 27-11-2015. 